\title{
Aspectos culturales de cepas nativas de Trichoderma spp. aislados de agroecosistemas de cacao nativo fino de aroma de la provincia de Bagua-Amazonas
}

\section{Cultural aspects of native strains of Trichoderma spp. isolated from fine aroma native cocoa agroecosystems of the province of Bagua-Amazonas}

\author{
Santos Triunfo Leiva Espinoza ${ }^{1 *}$ (D), Jaime Ramírez ${ }^{1}$, Christian Calle Yunis ${ }^{1}$ (D), Manuel Oliva ${ }^{1}$ (iD, Rolando Salas Lopez ${ }^{1}$ (D),
} Marielita Arce Inga ${ }^{2}$ (D), Alcides Román Peña ${ }^{1}$ (D), Malluri Goñas Goñas ${ }^{2}$ (D)

\section{RESUMEN}

El objetivo de la investigación fue evaluar los aspectos culturales de 12 cepas nativas de Trichoderma spp. Se llevó a cabo en un ambiente de laboratorio, donde se evaluó el crecimiento radial del micelio de 12 cepas nativas de Trichoderma spp., en tres diferentes medios de cultivo tales como: Agar Papa Dextroza , Zezapex Dox Agar y Dricloran Rosa de Bengala con tres temperaturas $\left(25^{\circ} \mathrm{C}, 30^{\circ} \mathrm{C}\right.$ y $\left.35^{\circ} \mathrm{C}\right)$. Se empleó un diseño completamente al azar con arreglo factorial, con 108 tratamientos y 3 repeticiones por cada uno, con un total de 324 unidades experimentales. Con los datos obtenidos se realizaron análisis de varianza, así mismo se efectuó pruebas de comparaciones múltiples de Tukey. Las cepas nativas de Trichoderma spp. (CP14-5 y CP10-3) fueron las que reportaron el mayor crecimiento radial de micelio con 3,79 cm y $3,69 \mathrm{~cm}$, respectivamente. El medio de cultivo en el que se reportó mayor crecimiento radial de micelio fue en el Agar Papa Dextroza con 3,78 cm. La temperatura de 30 ${ }^{\circ} \mathrm{C}$, fue en la que se mostró el mejor crecimiento radial del micelio de las cepas nativas de Trichoderma spp. con 3,24 $\mathrm{cm}$. Demostrando de esta menara la influencia que tiene la temperatura y medio de cultivo sobre el crecimiento radial del micelio de las cepas nativas de Trichoderma spp.

Palabras clave: antagonista, medio de cultivo, micelio, temperatura.

\begin{abstract}
The goal of the research was to evaluate the cultural aspects of 12 native strains of Trichoderma spp. It was carried out in a laboratory environment, where the radial growth of the mycelium of 12 native strains of Trichoderma spp. was evaluated, in three different culture media such as: Dextroza Potato Agar, Zezapex Dox Agar and Dricloran Rose Bengal with three temperatures $\left(25{ }^{\circ} \mathrm{C}, 30{ }^{\circ} \mathrm{C}\right.$ and $\left.35^{\circ} \mathrm{C}\right)$. A completely randomized design with factorial arrangement was used, with 108 treatments and 3 repetitions for each one, with a total of 324 experimental units. With the obtained data, analysis of variance was carried out, as well as multiple Tukey comparison tests. The native strains of Trichoderma spp. (CP14-5 and CP10-3) were the ones that reported the highest radial growth of mycelium with $3.79 \mathrm{~cm}$ and $3.69 \mathrm{~cm}$, respectively. The culture medium in which the greatest radial growth of mycelium was reported was Dextroza Potato Agar with $3.78 \mathrm{~cm}$. The temperature of $30^{\circ} \mathrm{C}$, was where the best radial mycelium growth of the native strains of Trichoderma spp. was shown with $3.24 \mathrm{~cm}$. Demonstrating from this menara the influence that the temperature and culture medium have on the radial growth of the mycelium of the native strains of Trichoderma spp.
\end{abstract}

Keywords: antagonist, culture medium, mycelium, temperature.

\footnotetext{
${ }^{1}$ Universidad Nacional Toribio Rodríguez de Mendoza de Amazonas, Instituto de Investigación para el Desarrollo Sustentable de Ceja de Selva, Chachapoyas, Perú

${ }^{2}$ Universidad Nacional Intercultural Fabiola Salazar Leguía de Bagua, Bagua

"Autor de Correspondencia, e-mail: santos.leiva@untrm.edu.pe
} 


\section{INTRODUCCIÓN}

El uso de microorganismos antagonistas de fitopatógeno habitantes del suelo, cobra cada vez más importancia ya que su aplicación no genera desequilibrios biológicos, y más bien regula o minimiza las poblaciones de fitopatógeno habitantes del suelo (Fajardo y Guzmán, 2006). Éste gran interés despertado por el control biológico de patógenos de plantas, es una respuesta en gran parte, a la creciente preocupación de la sociedad acerca del uso de agrodefensivos químicos (Chiriboga et al., 2015)

Schmoll et al. (2016) menciona que el género Trichoderma contiene hongos de gran relevancia para los seres humanos, con aplicaciones en la producción de enzimas para la degradación de la pared celular de las plantas y su uso en el control biológico. Es un hongo frecuentemente usado en actividades agrícolas como agente biocontrolador fúngico y un estimulante vegetal el cual surge como una alternativa al uso de agroquímicos, pues actúa como antagonista de diversas especies de hongos fitopatógenos (Hernández et al., 2011; Canonero et al., 2018). Este género posee múltiples mecanismos de acción, como la antibiosis, el micoparasitismo, la competencia por el espacio y los nutrientes y la producción de metabolitos secundarios (Hernández et al., 2019).

Este agente antagónico puede desarrollarse en una amplia gama de sustratos. Sin embargo, es probable que el hongo no responda igual a los diversos medios de cultivo en los que se siembre. El buen manejo del microorganismo en el laboratorio es fundamental para la mantener la capacidad de reproducción del hongo y no cambie ninguna característica fisiológica o corra el riesgo de sufrir alguna mutación que afecten el desarrollo de una investigación (Siordia et al., 2012).

En cuanto al efecto de la temperatura sobre el crecimiento micelial de Trichoderma, al ser un microorganismo mesófilo, su temperatura óptima de crecimiento es de 25 a $30^{\circ} \mathrm{C}$, pero a la vez es capaz de crecer en temperaturas que van de $\operatorname{los} 10$ a los $40{ }^{\circ} \mathrm{C}$. Esto pone en manifiesto que las temperaturas es un factor influyente en el crecimiento micelial de este hongo antagonista (Šimkovič et al., 2008).
En Perú, los estudios en la determinación de la temperatura y el medio de cultivo óptimo para el desarrollo de los microorganismos nativos in vitro son incipiente o simplemente no se han realizado. Esto genera una limitada información de las condiciones ambientales y del tipo de medio de cultivo que necesita un hongo asilado nativo para su buen crecimiento y desarrollo en el laboratorio. Todo ello, con la finalidad de que estas condiciones simulen que el microorganismo estuviese en el campo, y que su comportamiento y/o nivel de agresividad no varíe.

En este contexto, el objetivo de la investigación fue determinar los aspectos culturales de cepas nativas de Trichoderma spp. aislados de agroecosistemas de cacao nativo fino de aroma de la provincia de Bagua en la Región Amazonas de Perú

\section{MATERIALES Y MÉTODOS}

\section{Procedencia de las cepas nativas}

Se trabajaron con 12 de cepas nativas de Trichoderma spp., aislados de muestras de suelo de agroecosistemas de cacao nativo fino de aroma de la provincia de Bagua debidamente codificados (Tabla 1). Tras su recolecta, se llevaron las cepas nativas de Trichoderma spp. a los ambientes del Laboratorio de Investigación de Sanidad Vegetal (LABISANV) del Instituto de Investigación para el Desarrollo de Ceja de Selva (INDESCES) de la Universidad Nacional Toribio Rodríguez de Mendoza de Amazonas (UNTRM), para su conservación y determinación de los aspectos culturales.

Tabla 1. Cepas nativas de Trichoderma spp. evaluadas

\begin{tabular}{ccc}
\hline Distrito & Localidad & Cepas* \\
\hline Aramango & Pomabamba & CP61-1 \\
Copallín & Santa Ana & CP24-7 \\
Imaza & Pakum & CP10-3 \\
La Peca & San francisco & CP14-5 \\
Imaza & Nuevo Horizonte & CP4-3 \\
Copallín & Santa Ana & CP24-6 \\
La Peca & Arrayan & CP38-2 \\
Imaza & Pakum & CP11-3 \\
Copallín & Lluhuana & CP53-2 \\
La Peca & San Luis & CP27-1 \\
Aramango & Mirador & CP1-4 \\
La Peca & San Francisco & CP15-2 \\
\hline
\end{tabular}

*Cepas: CP61-1. (C: Cepa, P: Parcela, 61: Número de parcela y 1: Número de aislado) 


\section{Tratamientos de la investigación}

Se trabajó con un diseño completamente al azar (DCA) con un arreglo factorial. Se evaluaron 12 cepas nativas de Trichoderma spp., en tres medios de cultivo Agar Papa Dextroza (PDA), Zezapex Dox Agar (ZDA) y Dricloran Rosa de Bengala Agar (ARB) y tres temperaturas de cultivo 25,30 y $35^{\circ} \mathrm{C}$. Como resultado de la interacción de dichos factores, se obtuvieron 108 tratamientos con 3 repeticiones haciendo un total de 324 unidades experimentales evaluadas. Cada unidad experimental representó una placa petri de 9 cm de diámetro, con un área de 63,62 cm² (Tabla 2).

Tabla 2. Tratamientos de la investigación

\begin{tabular}{|c|c|c|c|c|c|c|c|c|c|c|c|}
\hline Tto* & Сера & $\begin{array}{l}\text { Medio } \\
\text { Cultivo }\end{array}$ & $\begin{array}{l}\text { Temp } \\
\left({ }^{\circ} \mathrm{C}\right)\end{array}$ & Tto* & Cepa & $\begin{array}{l}\text { Medio } \\
\text { Cultivo }\end{array}$ & $\begin{array}{l}\text { Temp } \\
\left({ }^{\circ} \mathrm{C}\right)\end{array}$ & Tto* & Сера & $\begin{array}{l}\text { Medio } \\
\text { Cultivo }\end{array}$ & $\begin{array}{l}\text { Temp } \\
\left({ }^{\circ} \mathrm{C}\right)\end{array}$ \\
\hline $\mathrm{T} 1$ & CP61-1 & PDA & 25 & T37 & CP4-3 & PDA & 25 & T73 & CP53-2 & PDA & 25 \\
\hline $\mathrm{T} 2$ & CP61-1 & PDA & 30 & T38 & CP4-3 & PDA & 30 & $\mathrm{~T} 74$ & CP53-2 & PDA & 30 \\
\hline T3 & CP61-1 & PDA & 35 & T39 & CP4-3 & PDA & 35 & $\mathrm{~T} 75$ & CP53-2 & PDA & 35 \\
\hline $\mathrm{T} 4$ & CP61-1 & ARB & 25 & $\mathrm{~T} 40$ & CP4-3 & ARB & 25 & T76 & CP53-2 & $\mathrm{ARB}$ & 25 \\
\hline T5 & CP61-1 & ARB & 30 & $\mathrm{~T} 41$ & CP4-3 & ARB & 30 & $\mathrm{~T} 77$ & CP53-2 & ARB & 30 \\
\hline T6 & CP61-1 & ARB & 35 & T42 & CP4-3 & ARB & 35 & T78 & CP53-2 & ARB & 35 \\
\hline $\mathrm{T} 7$ & CP61-1 & ZDA & 25 & T43 & CP4-3 & ZDA & 25 & T79 & CP53-2 & ZDA & 25 \\
\hline T8 & CP61-1 & ZDA & 30 & $\mathrm{~T} 44$ & CP4-3 & ZDA & 30 & T80 & CP53-2 & ZDA & 30 \\
\hline T9 & CP61-1 & ZDA & 35 & $\mathrm{~T} 45$ & CP4-3 & ZDA & 35 & T81 & CP53-2 & ZDA & 35 \\
\hline $\mathrm{T} 10$ & CP24-7 & PDA & 25 & T46 & CP24-6 & PDA & 25 & T82 & CP27-1 & PDA & 25 \\
\hline T11 & CP24-7 & PDA & 30 & T47 & CP24-6 & PDA & 30 & T83 & CP27-1 & PDA & 30 \\
\hline $\mathrm{T} 12$ & CP24-7 & PDA & 35 & $\mathrm{~T} 48$ & СР24-6 & PDA & 35 & T84 & CP27-1 & PDA & 35 \\
\hline T13 & CP24-7 & ARB & 25 & T49 & CP24-6 & $\mathrm{ARB}$ & 25 & T85 & CP27-1 & $\mathrm{ARB}$ & 25 \\
\hline T14 & CP24-7 & ARB & 30 & T50 & CP24-6 & ARB & 30 & T86 & CP27-1 & ARB & 30 \\
\hline $\mathrm{T} 15$ & CP24-7 & ARB & 35 & T51 & CP24-6 & ARB & 35 & T87 & CP27-1 & $\mathrm{ARB}$ & 35 \\
\hline T16 & CP24-7 & ZDA & 25 & T52 & CP24-6 & ZDA & 25 & T88 & CP27-1 & ZDA & 25 \\
\hline $\mathrm{T} 17$ & CP24-7 & ZDA & 30 & T53 & CP24-6 & ZDA & 30 & T89 & CP27-1 & ZDA & 30 \\
\hline T18 & CP24-7 & ZDA & 35 & T54 & CP24-6 & ZDA & 35 & Т90 & CP27-1 & ZDA & 35 \\
\hline T19 & CP10-3 & PDA & 25 & T55 & CP38-2 & PDA & 25 & T91 & CP1-4 & PDA & 25 \\
\hline $\mathrm{T} 20$ & CP10-3 & PDA & 30 & T56 & CP38-2 & PDA & 30 & T92 & CP1-4 & PDA & 30 \\
\hline $\mathrm{T} 21$ & CP10-3 & PDA & 35 & T57 & CP38-2 & PDA & 35 & T93 & CP1-4 & PDA & 35 \\
\hline $\mathrm{T} 22$ & CP10-3 & ARB & 25 & T58 & CP38-2 & $\mathrm{ARB}$ & 25 & T94 & CP1-4 & ARB & 25 \\
\hline $\mathrm{T} 23$ & CP10-3 & ARB & 30 & T59 & CP38-2 & $\mathrm{ARB}$ & 30 & T95 & CP1-4 & $\mathrm{ARB}$ & 30 \\
\hline $\mathrm{T} 24$ & CP10-3 & ARB & 35 & T60 & CP38-2 & $\mathrm{ARB}$ & 35 & T96 & CP1-4 & $\mathrm{ARB}$ & 35 \\
\hline $\mathrm{T} 25$ & CP10-3 & ZDA & 25 & T61 & CP38-2 & ZDA & 25 & T97 & CP1-4 & ZDA & 25 \\
\hline $\mathrm{T} 26$ & CP10-3 & ZDA & 30 & T62 & CP38-2 & ZDA & 30 & T98 & CP1-4 & ZDA & 30 \\
\hline $\mathrm{T} 27$ & CP10-3 & ZDA & 35 & T63 & CP38-2 & ZDA & 35 & T99 & CP1-4 & ZDA & 35 \\
\hline T28 & CP14-5 & PDA & 25 & T64 & CP11-3 & PDA & 25 & $\mathrm{~T} 100$ & CP15-2 & PDA & 25 \\
\hline T29 & CP14-5 & PDA & 30 & T65 & CP11-3 & PDA & 30 & $\mathrm{~T} 101$ & CP15-2 & PDA & 30 \\
\hline T30 & CP14-5 & PDA & 35 & T66 & CP11-3 & PDA & 35 & T102 & CP15-2 & PDA & 35 \\
\hline T31 & CP14-5 & ARB & 25 & T67 & CP11-3 & ARB & 25 & $\mathrm{~T} 103$ & CP15-2 & ARB & 25 \\
\hline T32 & CP14-5 & ARB & 30 & T68 & CP11-3 & $\mathrm{ARB}$ & 30 & T104 & CP15-2 & $\mathrm{ARB}$ & 30 \\
\hline T33 & CP14-5 & ARB & 35 & T69 & CP11-3 & ARB & 35 & T105 & CP15-2 & ARB & 35 \\
\hline T34 & CP14-5 & ZDA & 25 & T70 & CP11-3 & ZDA & 25 & T106 & CP15-2 & ZDA & 25 \\
\hline T35 & CP14-5 & ZDA & 30 & T71 & CP11-3 & ZDA & 30 & T107 & CP15-2 & ZDA & 30 \\
\hline T36 & CP14-5 & ZDA & 35 & $\mathrm{~T} 72$ & CP11-3 & ZDA & 35 & T108 & CP15-2 & ZDA & 35 \\
\hline
\end{tabular}

Tto*: Tratamiento.

\section{Crecimiento radial del micelio}

El crecimiento micelial se evaluó de acuerdo a la metodología de Dimbi et al, (2004), donde un fragmento de $5 \mathrm{~mm}$ de diámetro se obtuvo del borde de colonias de Trichoderma de 4 días de edad y se colocó en el centro de una caja de Petri con medio PDA. Las cajas se incu- baron a 25,30 y $35^{\circ} \mathrm{C}$. Se establecieron tres repeticiones por aislamiento y temperatura. El radio del crecimiento micelial se registró cada $24 \mathrm{~h}$. La prueba finalizó a los 3 días hasta que una de las colonias llenó la caja de Petri. Las medidas del último registro se utilizaron en el análisis estadístico. 
El crecimiento radial del micelio de las cepas de Trichoderma spp. (r, cm) se transformó en área de crecimiento $\left(\mathrm{A}, \mathrm{cm}^{2}\right)$ con la fórmula $\mathrm{A}=\pi \mathrm{r}^{2}$. El área correspondiente a cada crecimiento radial se expresó en porcentaje. Así, el efecto de la temperatura sobre el crecimiento micelial se evaluó mediante el porcentaje de inhibición e incremento del área, al pasar de 25 a 30 ${ }^{\circ} \mathrm{Cyde} 25$ a $35^{\circ} \mathrm{C}$.

Inhibición/incremento $(\%)=\frac{\text { Crecimiento } a 30^{\circ} \mathrm{C}}{\text { Crecimiento a } 25^{\circ} \mathrm{C}} \times 100$

Inhibición/incremento $(\%)=\frac{\text { Crecimiento } a 35^{\circ} \mathrm{C}}{\text { Crecimiento } a 25^{\circ} \mathrm{C}} \times 100$

\section{Análisis Estadístico}

Los datos tomados del crecimiento micelial de las 12 cepas nativas de Trichoderma spp. fueron analizados en el software estadístico Statistix versión 8, con el cual se realizó un análisis de varianza (ANOVA) al 5\% de significancia además se realizó una prueba de comparaciones múltiples de Tukey al $5 \%$, comparando las medias, tanto para el medio de cultivo como para la temperatura de incubación.

\section{RESULTADOS}

\section{Crecimiento radial del micelio de las cepas nativas de Trichoderma spp.}

El crecimiento micelial de las 12 cepas nativas de Trichoderma spp. mostraron diferencias significativas entre sí, obteniendo 9 grupos estadísticos (Tabla 3). El grupo estadístico A, obtuvo el mayor crecimiento micelial representado por la cepa CP14-5, que alcanzó un crecimiento micelial al tercer día de evaluación de 3,70 $\mathrm{cm}$. Sin embargo, no se observaron diferencias significativas con la cepa CP10-3 que alcanzo 3,69 cm. Por otro lado, las cepas que obtuvieron un crecimiento radial de micelio que oscilaron entre $2,73 \mathrm{~cm}$ hasta 1,88 cm fueron CP1-4, CP11-3, CP15-2 y CP4-3. Así mismo, el menor crecimiento radial del micelio lo conformo el grupo G, correspondiente a la cepa CP4-3, donde se obtuvo un crecimiento radial de $1,88 \mathrm{~cm}$, diferenciándose significativamente de las 11 cepas evaluadas.
Taba 3. Prueba de comparaciones múltiples para el crecimiento micelial de los 12 aislados

\begin{tabular}{ccc}
\hline Cepa & Media & $\begin{array}{c}\text { Grupos } \\
\text { Homogéneos }\end{array}$ \\
\hline CP14-5 & 3.70 & $\mathrm{~A}$ \\
CP10-3 & 3.69 & $\mathrm{~A}$ \\
CP61-1 & 3.57 & $\mathrm{AB}$ \\
CP38-2 & 3.39 & $\mathrm{ABC}$ \\
CP24-6 & 3.22 & $\mathrm{BCDD}$ \\
$\mathrm{CP} 24-7$ & 3.13 & $\mathrm{CDE}$ \\
$\mathrm{CP} 53-2$ & 2.88 & $\mathrm{DEF}$ \\
$\mathrm{CP} 27-1$ & 2.74 & $\mathrm{EF}$ \\
CP1-4 & 2.65 & $\mathrm{~F}$ \\
CP11-3 & 2.64 & $\mathrm{~F}$ \\
CP15-2 & 2.50 & $\mathrm{~F}$ \\
CP4-3 & 1.88 & $\mathrm{G}$ \\
\hline
\end{tabular}

Según la prueba de comparaciones múltiples de Tukey, se observaron tres grupos homogéneos (A, B y $\mathrm{C})$, dónde $\mathrm{A}$ es el medio de cultivo PDA, que reportó mayor crecimiento radial del micelio de los hongos evaluados. Mientras que el grupo C, representado por el medio de cultivo ZDA, fue el que obtuvo un menor crecimiento radial del micelio para las 12 cepas nativas de Trichoderma spp. evaluadas (Figura 1).

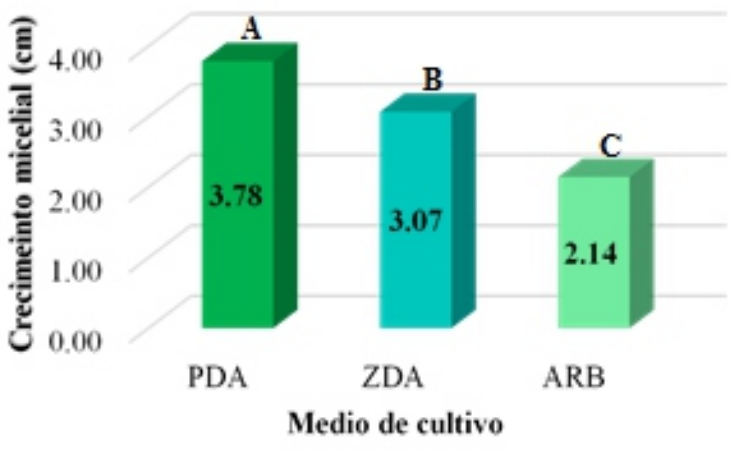

Figura 1. Prueba de comparaciones múltiples de Tukey para el tipo de medio de cultivo utilizado

En cuanto a las temperaturas, la prueba de comparaciones múltiples de Tukey determino la formación dos grupos homogéneos (A, B). Dónde el grupo A representa la temperatura dónde los hongos obtuvieron el mayor crecimiento micelial $\left(30^{\circ} \mathrm{C}\right)$, y el grupo $\mathrm{B}$ donde se obtuvo el menor crecimiento según la temperatura $\left(35\right.$ y $\left.25^{\circ} \mathrm{C}\right)$. 


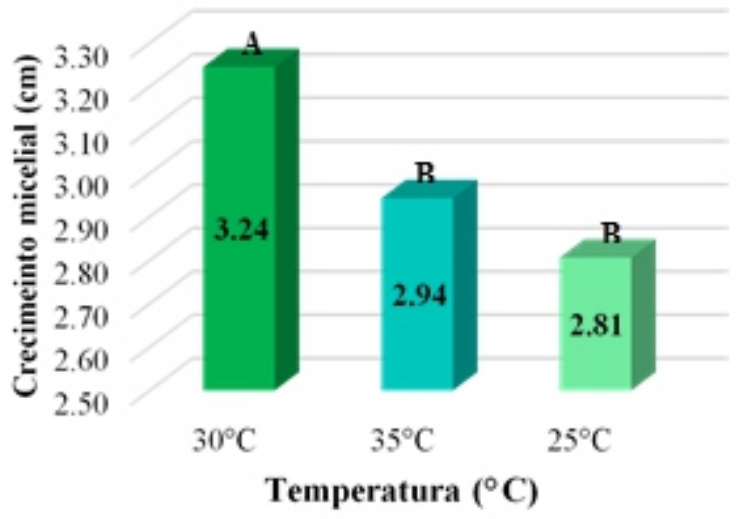

Figura 2. Prueba de comparaciones múltiples de Tukey para las temperaturas

\section{IV.DISCUSIÓN}

Al evaluar el efecto de las temperaturas de incubación in vitro en el crecimiento micelial de las cepas nativas de Trichoderma spp., se encontró que estos hongos se desarrollan con mayor facilidad a una temperatura de $30{ }^{\circ} \mathrm{C}$. Resultados poco similares a los de Victor et al. (2016) dónde encontraron que los aislamientos de Trichoderma crecieron a temperaturas entre 12 y 37 ${ }^{\circ} \mathrm{C}$, con un crecimiento máximo a $27^{\circ} \mathrm{C}$. Así mismo, estudios realizados por Maurya et al. (2017) encontraron que los hongos del género Trichoderma alcanzan el mayor crecimiento micelial a una temperatura entre $\operatorname{los} 25$ y $\operatorname{los} 30^{\circ} \mathrm{C}$. Por otro lado, Yadav y Chandra (2014) evaluaron el efecto de la temperatura para el crecimiento del hongo Pleurotus sp y encontraron que el hongo obtuvo un mayor crecimiento a $25^{\circ} \mathrm{C}$.

Con respecto al hongo que reportó el mayor crecimiento micelial (CP14-5) con 3,70 cm, proviene al distrito de la Peca. Éstos resultados pueden significar que dicho hongo tiene mayor facilidad de reproducción y puede que presente mayor agresividad en contra de los patógenos en comparación con las demás cepas evaluadas. Así mismo, se puede suponer que éste es un hongo con alta capacidad de adaptabilidad a diferentes temperaturas, considerando que al incubarse a una temperatura de 25,30 y $35^{\circ} \mathrm{C}$ el hongo reportó los mayores crecimientos radial del micelio (Fipke et al., 2015; Qui et al., 2017; Zehra et al., 2017).

En el caso de los medios de cultivo evaluados se encontró que el medio de cultivo más adecuado para el cultivo de las cepas de Trichoderma es el PDA, esto puede deberse al alto contenido en extracto de papa que favorece el crecimiento de los hongos en general. Hay investigaciones, que corroboran esta afirmación del PDA, ya que los valores más altos en crecimiento micelial de Trichoderma fueron encontrados en este tipo de agar (Jahan et al., 2013). Por otro lado, en el caso del medio de cultivo ARB, las cepas de Trichoderma comparado con el crecimiento en PDA obtuvieron menor crecimiento radial, esto pudo deberse a que éste medio de cultivo es específico para el control de levaduras y mohos en aire y superficie (Mentese et al., 2017). En el caso del medio ZDA, que también reportó un menor crecimiento, puede deberse a su alto contenido de sacarosa dentro de su composición. Esto hace que se la única fuente de carbono, mientras que el nitrato de sodio sirve como la única fuente de nitrógeno. Es probable que Trichoderma no necesita una fuente de carbono para su buen crecimiento y desarrollo (Kunanbayev et al., 2019).

\section{CONCLUSIONES}

Las cepas nativas de Trichoderma spp. CP14-5 y CP10-3, fueron las que reportaron el mayor crecimiento radial de micelio con $3,79 \mathrm{~cm}$ y $3,69 \mathrm{~cm}$, respectivamente.

El medio de cultivo que reportó mayor crecimiento radial de las 12 cepas nativas de Trichoderma spp fue PDAcon $3,78 \mathrm{~cm}$.

La temperatura en la que más crecimiento micelila de Trichoderma, se dio a $30^{\circ} \mathrm{C}$, con un promedio de crecimiento radial de micelio de $3,24 \mathrm{~cm}$.

\section{REFERENCIAS BIBLIOGRÁFICAS}

Canonero L. J., F. Lattanzi, M. M. Molina, y L. S. Pinotti. 2018. Evaluación de la capacidad biocontroladora de Trichoderma sp. en un cultivo de garbanzo (Cicer arietinum) en la región semiárida de la provincia de Córdoba. Tesis de Grado. Universidad Nacional de Cordova. Cordova (Argentina).

Chiriboga, H., G. Gómez, K. Garcés. 2015. Protocolos para formulación y aplicación del bio- 
insumo: Trichoderma spp. ara el control biológico de enfermedades. Asunción (Paraguay): Instituto Interamericano de Cooperación para la Agricultura (IICA)

Dimbi S., N. K. Maniania, S. A. Lux, y J. M. Mueke. 2004. "Effect of constant temperatures on germination, radial growth and virulence of Metarhizium anisopliae to three species of African tephritid fruit flies." BioControl 49 (1): 83-94.

Fajardo P. C. y A. M. Guzmán. 2006. "Cultivo in vitro de Trichoderma spp. su antagonismo frente a Hongos Fitopatógenos." Revista ESPAMCIENCIA (1):21-28

Fipke, G. M., J. B. Pazini, y L. Z. Ethur. 2015. “Antagonismo de isolados de Trichoderma spp. ao Sclerotinia sclerotiorum em diferentes temperaturas." Magistra 27 (1):23-32

Hernández D. J., R. Ferrera, y A. Alarcón. 2019. “Trichoderma: Importancia agrícola, biotecnológica, y sistemas de fermentación para producir biomasa y enzimas de interés industrial." Chilean Journal of Agricultural \& Animal Sciences 35 (1): 98-112.

Hernández J. L., S. Pérez, M. Isabel, J. G. García, N. Mayek, y J. M. González Prieto. 2011. "Molecular and agronomic characterization of Trichoderma spp. natives of northeastern Mexico." Revista Colombiana de Biotecnología 13 (2): 176-185.

Jahan N., S. Sultana, S. K. Adhikar, S. Rahman, y S. Yasmin. 2013. "Evaluation of the growth performance of Trichoderma harzianum (Rifai.) on different culture media." Journal of Agriculture and Veterinary Science 3 (4): 44-50.

Kunanbayev, K., G. Churkina, I. Rukavitsina, N. Filippoca, y M. Utebayev. 2019. "Potential attractiveness of soil fungus Trichoderma inhamatum for biodegradation of the glyphosate herbicide." Journal of Ecological Engineering 20 (11): 240-245.
Maurya M. K., M. Srivastava, A. Singh, S. Pandey y V. Ratan. 2017. "Effect of different temperature and culture media on the mycelia growth of Trichoderma viride isolates." International Journal of Current Microbiology and Applied Sciences 6(2): 266-269.

Mentese, S., M. T. Otkun, y E. Palaz. 2017. “Comparison of dichloran rose bengal chloramphenicol and Sabouraud dextrose agar with cycloheximide and chloramphenicol for airborne mold sampling." Aerobiologia 33: 211219.

Qui, Z., X. Wu, J. Zhang, y C. Huang. 2017. "High temperatura enhances the ability of Trichoderma asperellum to infect Pleurotus ostreatus mycelia." PLoS ONE 12 (10): e018755.

Schmoll M., C. Dattenboeck, N. Carreras, A. Mendoza, D. Tisch, M. Alemán, y G. Cristobal. 2016. "Los genomas de tres hermanos desiguales: huellas de los estilos de vida de tres especies de Trichoderma." Microbiol. Mol. Biol. Rev 80 (1): 205-327.

Šimkovič M., P. Ditte, A. Kurucova, B. Lakatoš, y L. U. Varečka. 2008. " $\mathrm{Ca}^{2+}$-dependent induction of conidiation in submerged cultures of Trichoderma viride." Canadian Journal of Microbiology. 54 (4):291-298.

Siordia M. A., A. Figueroa, y H. M. Cárdenas. 2012. "Efecto de los Componentes de seis medios de cultivo solido sobre la velocidad de crecimiento de Trichoderma harzianunm y su Producción." En XII Congreso Nacional de Biotecnología y Bioingeniería. Sinaloa (México).

Victor M., P. F. Domingues, K. Elaine De Moura, D. Salomão, L. M. Elias, F. Rodrigues, A. Patrício. 2016. "Effect of temperature on mycelial growth of Trichoderma, Sclerotinia minor and S. sclerotiorum, as well as on mycoparasitism." Summa Phytopathol 42 (3): 222-227. 
Yadav M. K., y R. Chandra. 2014. "Evaluation of culture media, $\mathrm{pH}$ and temperature for mycelial growth of different strains of Pleurotus sp." Agricultural Science Digest - A Research Journal 34 (4): 299-302.

Zehara, A., M. K. Dubey, M. Meena, y R. S. Upadhyay. 2017. "Effect of different environmental conditions on growth and sporulation of some Trichoderma species." Journal of Environmental Biology 38 (2): 197-203 